\title{
Advanced Control of Thermostatic Loads for Rapid Frequency Response in Great Britain
}

\author{
Vincenzo Trovato, Member, IEEE, Inmaculada Martínez Sanz, Member, IEEE, \\ Balarko Chaudhuri, Senior Member, IEEE, and Goran Strbac, Member, IEEE
}

\begin{abstract}
In the Great Britain power system, reduced system inertia (particularly during low demand conditions) and larger possible infeed loss would make grid frequency regulation extremely challenging in future. Traditional primary frequency response could be insufficient to limit the frequency variation within acceptable range. This paper shows that thermostatically controlled loads (TCLs) (domestic refrigerators) can be controlled without real-time communication and in a non-disruptive way to collectively enhance the network frequency response. The aggregated power consumption of TCLs, distributed across the system, could be controlled as a 'linear' function of the locally measured frequency and its rate of change. Alternatively, their aggregated consumption could be made to follow a 'pre-set' power profile depending on the estimated infeed loss. A novel technique for accurate estimation of infeed loss and consequent post-fault TCL power reduction is also proposed. The effectiveness of the two TCL control strategies are compared for primary and secondary frequency response through a case study on a 36 busbar reduced equivalent of the Great Britain power system. The effect of spatial variation of transient frequencies and the time delays in frequency measurement and filtering are considered to show how the TCLs can realistically provide rapid frequency response.
\end{abstract}

Index Terms-Frequency Response, Thermostatic Loads, Power System Dynamics, Demand-side Response.

\section{INTRODUCTION}

$\mathbf{I}$ N Great Britain (GB), the system operator, National Grid (NG), is responsible for maintaining the transient frequency deviation within $49.2 \mathrm{~Hz}$ following large infrequent infeed losses [1]. During grid frequency variations, generators and frequency-sensitive loads respond to address the demandsupply mismatch caused by an infeed loss. The amount of frequency response required at a particular time depends primarily on the system inertia, the largest possible infeed loss and the speed of frequency response provision.

In future, a significant proportion of electricity in GB is expected to be generated from on- and off-shore wind farms and other forms of Renewable Energy Sources (RES) [2]. Wind farms and most other RES use non-synchronous generators (NSGs) which do not intrinsically contribute to system inertia [3] unlike traditional synchronous machines. During low demand conditions, in particular, there would be less number of synchronous generators in operation (RES

This work was supported by the Engineering and Physical Science Research Council, U.K., under grants EP/K036327/1 and EP/L001039/1. Supporting data is available on request: please contact cappublications@imperial.ac.uk.

The authors are with the Control and Power Research Group, Department of Electrical and Electronic Engineering, Imperial College London, London, SW7 2AZ, U.K. (e-mail: v.trovato10@imperial.ac.uk) typically have merit order priority) which could result in significant reduction in system inertia with less provision for traditional frequency response [2]. For these reasons, limiting the frequency nadir within $49.2 \mathrm{~Hz}$ would require additional 'conventional' frequency response (acting in ten seconds [1]) which would increase operational costs. By 2020, NG envisages a $30-40 \%$ increase in frequency response requirements compared to the 2015 levels. This value is expected to rise up to $300-400 \%$ by 2030 [2]. However, the amount of frequency response required to ensure acceptable frequency deviation could be reduced if the speed of response is increased. This has prompted NG to announce a new market for Enhanced Frequency Response (EFR) providers, which are meant to respond within one second [4]. Alongside options like energy storage, interconnectors, etc., fast demand-side response (DSR) could be a cost-effective candidate to maintain the network frequency above security thresholds and reduce the additional amount of frequency response required from conventional sources. Recent studies have demonstrated the DSR concept from distributed resources [5]-[8] although without directly referring to a specific demand-side technology. The particular load dynamics and control requirements would play a role in the actual application of these schemes.

Thermostatically Controlled Loads (TCLs), like refrigerators, air-conditioners, etc., are a potential candidate for flexible DSR. Under certain boundaries, the regular operating cycle of these devices can be altered without noticeable impact on the controlled temperature. Because of the large number of TCLs connected to the grid and their relative insensitivity to temperature fluctuations, the TCLs could potentially entail a more cost-effective power system operation by providing various forms of ancillary services [9]-[11]. However, control of individual devices for network service provision presents several challenges which need to be overcome before the system operators could rely on them [12].

Considering the TCL ability to provide frequency response, the performance of a TCL controller largely depends on its capability to respond to frequency events within seconds and to execute a dependable recovery to steady-state operation. The controller ideally should not rely on real-time communication, which requires an expensive infrastructure (due to the large number of TCLs) and is vulnerable to communication failures. Moreover, in accordance with the recommendations of the ENTSOe Demand Connection Code [13], the controller has to be strictly non-disruptive i.e. individual TCLs should not ever exceed upper and lower temperature thresholds, while providing a service to the system. 
Decentralized control strategies for the provision of frequency response from TCLs have been reported in [14]-[20]. Although these solutions do not require real-time communication, they tend to suffer from sustained oscillations in TCL power consumption. In addition, single-device temperatures may not be limited within the nominal range. These problems are somewhat addressed by the control strategies reported in [21] and [22]. The first strategy prevents device-level temperature excursions, while the second one guarantees a non-oscillating TCL recovery pattern, although impeding rapid load changes. An alternative approach is described in [23] where a real-time controller dispatches frequency-sensitive price signals to enable frequency response provision from the appliances.

Another class of TCL controllers aim at the accurate tracking of pre-defined power profiles [24]-[28]. This has been used for providing long term support to the power system (e.g. energy arbitrage [24]). The extension of the control strategies in [24]-[27] to fast frequency response applications is limited by the centralized real-time communication infrastructure required over short timescales. The control framework for TCLs proposed in [28] accurately derives the desired power response through both deterministic and stochastic switching of the power states of individual TCLs. The control strategy is fully decentralized and it strictly respects the upper and lower temperature constraints of individual TCLs.

The first contribution of this paper is the detailed extension of the general control scheme described in [28] for the tracking of generic power profiles. This paper enables the controller for fast frequency response provision while addressing in detail specific implementation requirements. Two different controllers are introduced. First, a Linear Controller (LC) is used to make the aggregate power consumption of TCLs always a linear function of the locally measured frequencies and their rates of change (RoCoF). This property was not guaranteed in previous works (e.g. [14]). Then, a Pre-Set Shape Controller (PSSC) is employed to follow a pre-defined reference power profile depending on the estimated infeed loss. Although the general control framework in [28] is fully decentralized, realistic and effective power system applications proposed in this paper may require a communication infrastructure. Crucially, a low-latency channel is still not necessary. For both control strategies, TCL response is activated once the locally measured frequency or RoCoF are outside the specified thresholds [29], [30]. The time lag between activation of TCL response at different busbars and the time delays associated with frequency measurement and filtering are considered in the study to provide a realistic measure of the expected frequency response contribution from the TCLs.

The second contribution of this paper is proposing a novel technique for accurate estimation of infeed loss, which is critical while using a PSSC. This technique not only estimates the infeed loss accurately similar to the technique reported in [31], but it also ensures the desired post-fault reduction in aggregate power consumption of the distributed TCLs.

The performance of TCL support has to be validated by the system operator in a realistic multi-machine power system model before considering TCLs a reliable source of response services. In addition, the effect of spatial variation in inertia, which could be pronounced in the GB system in future [2], cannot be captured through an equivalent single-machine representation. Due to spatial variation of inertia, the transient frequency evolution immediately after a large infeed loss could vary significantly between different busbars/regions. These different frequency dynamics depend not only on the aggregated inertia at a busbar/region but also on its electrical proximity to the neighboring sources of inertia [32] affecting the ability of TCLs to contribute to frequency response locally. In face of this, it is critical to ensure a coherent activation of TCL response which can only be demonstrated in a multi-machine framework. However, it is virtually impossible to simulate the individual power-temperature dynamics of millions of TCLs within a multi-machine transmission and distribution network model. The potential benefit of dynamic control of intelligent loads in a multi-machine system has been covered in [8]. In this case the challenges associated with aggregating and implementing a real control infrastructure for individual devices to generate a desired aggregated response were not addressed properly, albeit other simplifying assumptions. Similarly, in [21], the distributed TCL contribution is demonstrated considering aggregate loads which do not accurately take into account device-level dynamics. In [20] the number of simulated devices is limited and requires two different simulation softwares.

The third contribution of this paper is the demonstration of the effectiveness of individual distributed TCLs towards providing rapid frequency response through a case study on a 36 busbar reduced equivalent of the GB power system. Note that 'rapid' refers to the ability of TCLs to provide the full amount of primary response within a time frame (one second) smaller than the typical response time (ten seconds) required from conventional generators in GB [1]. Based on the general control properties in [28], the extension of the proposed controllers (LC and PSSC) to the multi-machine system is compared at a system level and validated over different response time-scales (primary and secondary). It is worth mentioning that the control framework in [28] was applied, as a first step, to an equivalent single machine system in [33]. Several simplifying assumptions like ideal continuous frequency measurements, absence of time delays and finite response times were made which were far from practical. These limitations have been overcome in this paper.

The structure of the paper is as follows. Section II recalls the basics of the TCL control framework in [28] to manage the TCL aggregate power consumption while Section III describes the common settings for the presented TCL controllers in order to provide frequency services. The proposed LC and PSSC controllers are described, respectively, in Sections IV and V. The case study corresponding to the GB transmission is introduced in Section VI and simulation results appear in Section VII. Finally, Section VIII presents conclusions and future research directions.

\section{BASICS OF TCL CONTROL STRATEGY}

A decentralized deterministic-stochastic control strategy to manage the aggregate power consumption of a cluster of TCLs 
was proposed in [28]. The control strategy is described by (1).

$$
P(t)=P_{0} \cdot \Pi(t)+O\left(N^{-1 / 2}\right)
$$

The aggregate steady-state power consumption $P_{0}[\mathrm{MW}]$ of a population of $N$ TCL devices is modulated by a reference power profile $\Pi(t)$ to control the aggregate instantaneous power $P(t)$ [MW]. In steady-state this results in $\Pi(t)=\Pi_{0}=1$. It has to be noted that $O\left(N^{-1 / 2}\right)$ represents the relative deviations from the expectation, which decrease approximately as $1 / \sqrt{N}$. The accuracy of the power modulation therefore increases with the number of TCLs. The control law (1) is valid for the general case of heterogeneous devices [28]. However, for the reminder of this paper, identical TCLs have been considered for simplicity without loss of generality. The reference power profile $\Pi(t)$ and the average temperature $\bar{T}(t)$ of the TCL cluster are related as:

$$
\frac{d \bar{T}(t)}{d t}=-\frac{1}{\tau}\left(\bar{T}(t)-T_{o f f}+\Pi(t) \cdot\left(T_{o f f}-\bar{T}_{0}\right)\right)
$$

where $\tau[\mathrm{s}]$ is the thermal time constant of TCLs, $T_{o f f}\left[{ }^{\circ} \mathrm{C}\right]$ the ambient temperature and $\bar{T}_{0}\left[{ }^{\circ} \mathrm{C}\right]$ the steady-state average temperature. Note that (2) holds for the common case of TCLs modeled by linear first-order ordinary differential equations representing the thermal dynamics. It is demonstrated in [28] that, if the system-level power/temperature constraints (3)-(4) are satisfied, a power response $P(t)$ can be accurately tracked in a decentralized way by the individual TCLs without letting any TCL ever deviate from their specified temperature limits $\left[T_{\min }, T_{\max }\right]$. This implies that device-level simulations are not necessary if (3)-(4) are satisfied.

$$
\begin{aligned}
P_{\min }=P_{0} \cdot \Pi_{\min } & \leq P(t) \leq P_{\max }=P_{0} \cdot \Pi_{\max } \\
T_{\min } & \leq \bar{T}(t) \leq T_{\max }
\end{aligned}
$$

As the TCL controller guarantees the respect of upper/lower temperature thresholds for individual devices, it can be classified as a non-disruptive controller. The expressions for $\Pi_{\max }$ and $\Pi_{m i n}$, which are constant quantities only depending on thermal model parameters of TCLs, can be found in [34]. The limits on the reference power profile (3) prevent instantaneous power excursions while (4) in combination with (2) avoids any sustained power profile that would result in temperature violation of individual TCLs.

Monte Carlo simulations of individual device actions are used to demonstrate the general results of the control strategy (1)-(3-4). The following illustrative example simulates the behaviour of 60000 appliances, which are initialized with random initial temperatures. The default parameters refer to domestic refrigerators as in [34]. The devices are instructed to track an arbitrary reference power curve $\Pi(t)$ (black traces in Fig. 1 (a)). The empirical power consumption of the simulated appliances is shown in red traces in Fig. 1 (a), confirming the accurate tracking of the desired power profile and the feasibility of the solution. Note that in Fig. 1 (a), the Yaxis is limited to 1.5 although $\Pi_{\max }$ is, in compliance with (3), a larger quantity. Fig. 1 (b) shows the temperatures of 100 simulated devices during the tracking of the reference power signal. It is clear that the controller is indeed nondisruptive. Note that the parameters of individual TCLs have
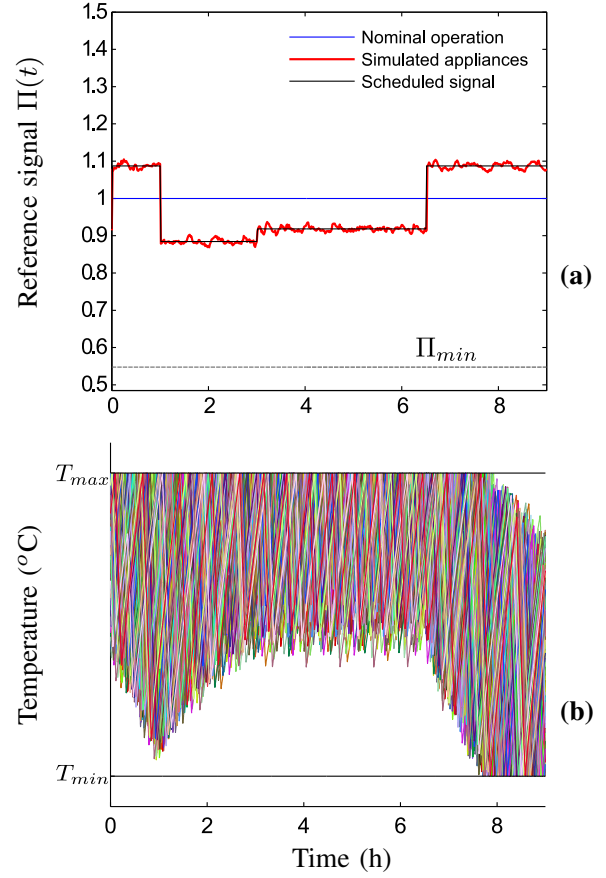

Fig. 1. Device-level validation of TCL control strategy: (a) Power tracking capability; (b) Individual temperature variations during power reference tracking.

been independently varied by $\pm 15 \%$ to generate a set of heterogeneous appliances. Temperatures in Fig. 1 (b) are therefore shown on a scale between $T_{\min }$ and $T_{\max }$ to account for heterogeneity of the TCL population. The results show that a coherent response does not require the use of identical appliances.

\section{Frequency Response Provision from TCLs}

In [28] the appliances aimed to deliver a predetermined power response $\Pi(t)$ without a well-defined activation methodology and without taking into account specific power system applications. For instance, the presented response was not tailored for frequency service provision. This paper elaborates a more general framework for TCL control as the aggregate TCL power trajectory is specifically designed for frequency control. In addition, the response is based on the particular system operating scenario and the TCL geographical location in the system. The model (1)-(3-4), describing the dynamics of a population of TCLs, is compact. This property has been exploited in this work to directly integrate the power/temperature response of millions of TCLs within a large-scale power system simulation platform (e.g. DIgSILENT PowerFactory) [35].

Two control strategies are presented in this paper to extract frequency response from TCLs - the Linear Controller (LC) and the Pre-Set Shape Controller (PSSC). The reference power profile $\Pi(t)$ is determined in a different way for each control strategy as discussed later in Sections IV and V. Frequency response services over different time scales are considered which include primary (10-30 seconds) and secondary (up to 30 minutes) responses in line with National Grid (NG) specifications [1], [4]. In this paper, only the transmission 
network is modeled neglecting the frequency dynamics at the distribution level. All loads (including the TCLs) are aggregated at each transmission level busbar and would experience the same frequency dynamics if connected to the same busbar.

\section{A. Activation of TCL Response}

The aggregate response from TCLs connected to the busbar $i$ is activated if one of the following conditions is violated:

$$
\Delta f_{i}>\Delta f_{\text {limit }} \vee \mathrm{RoCoF}_{i}>\mathrm{RoCoF}_{\text {limit }}
$$

Frequency is measured locally at each busbar $i$ with $20 \mathrm{~ms}$ sampling according to the minimum frequency measurement rate required by the ENTSOe Demand Connection Code [13]. The response from TCLs is activated when the resulting filtered frequency deviation from nominal value $f_{0}$, $\Delta f_{i}=f_{0}-f_{i}$, exceeds the $\Delta f_{\text {limit }}= \pm 0.2 \mathrm{~Hz}$ threshold [29]. The second criterion is based on the maximum threshold for the rate of change of frequency $(\mathrm{RoCoF})[\mathrm{Hz} / \mathrm{s}]$ measured over a sliding time window $\Delta t_{s}$ as defined in (6).

$$
\begin{array}{r}
\operatorname{RoCoF}_{i}\left(t, \Delta t_{s}\right)=\frac{f_{i}\left(t-\Delta t_{s}\right)-f_{i}(t)}{\Delta t_{s}} \\
\frac{d f_{i}(t)}{d t}=\lim _{\Delta t_{s} \rightarrow 0} \operatorname{RoCoF}_{i}\left(t, \Delta t_{s}\right)
\end{array}
$$

It is important to note the distinction between $\mathrm{RoCoF}$ and frequency derivative in (6) and the $\mathrm{RoCoF}$ dependence on the size of the sliding time window. For the remainder of this paper, RoCoF values are calculated over different $\Delta t_{s}$, according to the needs. The $R_{o} \mathrm{CoF}_{i}$ calculation for the TCL response activation in (5) employs a sliding window of $500 \mathrm{~ms}$. An upper threshold of $R o C o F_{\text {limit }}=1 \mathrm{~Hz} / \mathrm{s}$ has been used according to the proposed loss of mains protection settings for distributed generators in future GB low inertia scenarios [30]. Once the aggregate TCL response is activated at each busbar $i$, the individual TCL controllers will track the reference power profile $\Pi_{i}(t)$ in a decentralized way. An additional $200 \mathrm{~ms}$ time lag is included in the control loop to consider the impact of any unaccounted source of delay.

\section{B. Autonomous vs. Semi-autonomous Operation of TCLs}

If the desired power profile $\Pi(t)$ is to be broadcasted in realtime to all the appliances, a dedicated communication channel would be required. The speed of the signal transmission would be therefore crucial. Such a communication infrastructure entails high costs and is sensitive to disruptions. On the other hand, the control of TCLs could be exercised in a fully autonomous mode where distributed TCLs can independently construct and follow a reference power profile $\Pi(t)$ based on the locally available signals (e.g. frequency, time), as in [28]. However, in the context of frequency support from distributed appliances, the aggregate TCL response would remain the same regardless of the severity of the infeed loss event and the frequency deviation and its rate of change. The risk of underor over-response from the TCL population rises, affecting the demand-side flexibility and controllability [28].

Alternatively, as envisaged in [28], a semi-autonomous mode could better exploit the TCL support by making use of a non-time critical communication channel. In this framework, the TCL response is updated periodically (for example, every hour) depending on the actual requirements at a given point in time. For instance, this would depend on the level of inertia, the system demand and the governor characteristics of the online generators. This way under- or over-response from the TCLs is avoided or minimized and the system operator can schedule response services accordingly. The updates are required on much slower time scale (hourly or more) compared to the response time of TCLs (seconds to minutes). Hence, the smart metering infrastructure [36] would be adequate rather than requiring dedicated real-time communication with TCLs (as for centralized controllers).

The following sections present the implementations of two control schemes for TCL frequency response provision. Benefits and requirements introduced by the semi-autonomous operation of the TCL controllers are also discussed. According to this, it is assumed that a centralized entity is in charge of selecting and updating the TCL aggregate response. Moreover, for simplicity, it is also assumed that such a demand-response aggregator, which could be the Transmission System Operator (TSO), has perfect knowledge of the devices capabilities i.e. it knows the parameters of the TCL thermal models. Given this knowledge, the TSO is able to ensure that the desired profile $\Pi(t)$ can be realized by the appliances without violating their quality of service requirements. Moreover, this allows to verify that, for a particular system scenario, the chosen TCL response does not lead to over/under power modulations. It is worth pointing out that when this assumption is removed, simple and effective means to assess the compatibility of the device parameters and any proposed response curve, based on a set of aggregate parameters, can be determined following [28], [34].

Finally, alongside frequency response services, a semiautonomous operation could be used for energy arbitrage where the TCLs could be controlled to alter their power consumption and exploit the energy price differences over a certain time window [13]. This study does not aim to determine the optimal shape of the TCL response based on frequency service provision alone. The optimal services allocation would also be driven by further system-level technical, economic and policy considerations [13].

\section{The Linear Controller (LC)}

The Linear Controller (LC) determines the reference power profile for the TCLs according to the control law in (7).

$$
\Pi_{i}^{L}(t)=1-K_{1} \cdot \Delta f_{i}(t)-K_{2} \cdot \operatorname{RoCoF}_{i}\left(t, \Delta t_{s}\right)
$$

Note that, for under-frequency events, $\Delta f_{i}(t)=f_{0}-f_{i}$ is always a positive quantity, while $\operatorname{RoCoF}_{i}\left(t, \Delta t_{s}\right)$ may change sign during the frequency transient. The aggregate power consumption of the TCLs $P_{i}(t)=P_{0_{i}} \cdot \Pi_{i}^{L}(t)$ at busbar $i$ is thus a linear function of the local frequency deviation $\Delta f_{i}(t)$ (with a factor $K_{1}$ ) and its rate of change $\mathrm{RoCoF}_{i}$ (with a factor $K_{2}$ ), calculated over $20 \mathrm{~ms}$. The values of $K_{1}$ and $K_{2}$ are chosen such that when $\Delta f_{i}(t)$ and $R o C o F_{i}$ achieve minimum thresholds the reference power level equals $\Pi_{\text {min }}$. 
This ensures maximum contribution from the TCLs for these conditions.

$$
\begin{aligned}
K_{1} \mid \Pi^{L}\left(\Delta f_{m i n}\right) & =\Pi_{m i n} \quad \rightarrow \quad K_{1}=\frac{\Pi_{0}-\Pi_{m i n}}{\Delta f_{m i n}} \\
K_{2} \mid \Pi^{L}\left(R o C o F_{m i n}\right) & =\Pi_{m i n} \quad \rightarrow \quad K_{2}=\frac{\Pi_{0}-\Pi_{m i n}}{R o C o F_{m i n}}
\end{aligned}
$$

We consider $\Delta f_{\min }$ equal to $0.8 \mathrm{~Hz}$ [29] and $R o C o F_{\min }$ equal to $1 \mathrm{~Hz} / \mathrm{s}$ [30]. $K_{1}$ and $K_{2}$ are constant quantities (they do not depend on measured signals e.g. frequency) as defined above and equal for all the TCL clusters. Although the design of $K_{1}$ and $K_{2}$ in (8) does not directly take into account the actual system operating condition, an effective TCL response can be obtained, in general, for different scenarios. In fact, it is demonstrated in Section VII-C that the proposed LC does not lead to under- or over-response from the TCL cluster. Nevertheless, if required, the device support can be still updated periodically (i.e. modifying $K_{1}$ and $K_{2}$ ) under the semi-autonomous operation discussed earlier. Generalization of this for heterogeneous TCL populations is straightforward. In particular, $\Pi_{0_{i}}=\Pi_{0}=1$, by construction, while, $\Pi_{m i n_{i}}$, the minimum reference power level of the heterogeneous population of TCLs at busbar $i$, would equal $\max _{k} \Pi_{\text {min }_{i}}^{k}$, the maximum of the minimum reference power levels among all the $N_{i}$ TCLs included in the cluster. The LC (7) at each busbar ensures that the aggregate TCL power consumption satisfies the constraint (3) at all times. Hence, if either the frequency deviation or the RoCoF are outside the specified threshold, the aggregate TCL consumption would be constant at $P_{\text {min }_{i}}=P_{0_{i}} \cdot \Pi_{m i n}$. Temperature limits in (4) are also enforced and can be monitored by integrating (2).

The proposed LC also enables the TCL provision of secondary frequency response. During such a relatively long time interval $(30 \mathrm{~min})$, the aggregate TCL power consumption still follows the control law (7) until the frequency is completely restored. As constraints (3) and (4) are satisfied, the device level feasibility of the power response is guaranteed.

\section{The Pre-Set Shape Controller (PSSC)}

The Pre-Set Shape Controller (PSSC) tracks a reference power profile $\Pi^{P}(t)$ with predefined shape. Here we use the simplest possible form which is a controllable constant power reduction with respect to the initial steady-state power consumption of the TCLs. Limits on possible extent of power reduction are set by (3) and (4), and applied to primary and secondary response time intervals. The same power reduction level is sustained over the primary and secondary response time frame, which maximizes the TCL contribution in response to a severe frequency event. The TCL response is activated by the frequency- and RoCoF-based criteria (described in Section III-A) but the amount of response does not depend on the measured frequency signals and/or RoCoF unlike the LC. The amount of power reduction is determined by the estimated level of infeed loss to avoid under- or over-response from the TCLs as described next.

\section{A. Estimation of Infeed Loss}

Accurate estimation of infeed loss is critical for satisfactory operation of the PSSC. In a multi-machine power system, the frequency dynamics at each busbar immediately after the event depend on the local inertia (connected at the busbar) as well as the level of inertia at (electrically) nearby busbars. Ultimately, electrical proximity depends on the effective reactance between the busbars [32]. Therefore, for infeed loss estimation, we propose to divide the network into $N_{a}$ coherent areas in which the initial transient frequency dynamics are similar. Assuming that each area $j$ contains busbars $i=1, \ldots, n_{j}$, individual TCL would estimate a proportion of the infeed loss locally as:

$$
\left(\tilde{P}_{j}^{\text {loss }}\right)_{i}=2 \frac{H_{j}}{f_{0}} \frac{\Delta f_{i}}{\Delta t}
$$

where, $H_{j}$ is the aggregate inertia of all the $i=1 \ldots n_{j}$ busbars within area $j \in N_{a}$. This way, the estimated infeed loss is similar for all the TCLs within an area. The coherent areas could be determined offline through the simulated trends in bus frequencies across the system. For improved accuracy, the frequency deviation $\Delta f_{i}$ is evaluated in the first $500 \mathrm{~ms}$ after the frequency event or within the time when $\Delta f_{i}$ exceeds $\Delta f_{\text {limit }}$, whichever occurs first. The total system-wide infeed loss could be estimated by averaging the infeed loss at each busbar:

$$
\tilde{P}_{\text {sys }}^{\text {loss }}=\sum_{j=1}^{N_{a}}\left[\frac{1}{n_{j}} \sum_{i=1}^{n_{j}}\left(\tilde{P}_{j}^{\text {loss }}\right)_{i}\right]
$$

Remark 1: It is worth pointing out that the size of the infeed loss is estimated in order to extract the correct amount of response from the distributed TCLs. This is key for comparing our estimation approach against the ones reported in literature. In [31], the local proportion of the infeed loss was calculated at each busbar $i$ using the local inertia $H_{i}$. Although this method offers an accurate average estimation of overall the infeed loss (summing the proportion of infeed loss across all the busbars), a TCL control strategy based on this estimation might activate insufficient TCL response. This is because the infeed loss estimation at each busbar could vary significantly depending on the inertia at each busbar. Therefore, the TCL response capability at busbars with low inertia could be underutilized and vice versa. In the extreme (but realistic) case, the local inertia at the busbars where no generators are connected would be zero and the TCLs connected to those busbars would not respond. With the proposed methodology, the TCLs within an area would estimate similar infeed loss and derive similar reference power reduction which is ultimately proportional to the TCL penetration at each busbar.

\section{B. The Control Law}

According to the estimated infeed loss $\left(\tilde{P}_{j}^{\text {loss }}\right)_{i}$, the reference power profile for each TCL is defined as:

$$
\Pi_{i}^{P}(t)= \begin{cases}\Pi_{\min } & \left(\tilde{P}_{j}^{\text {loss }}\right)_{i} \geq P_{j}^{R_{\max }} \\ \Pi_{0}-\frac{\left(\tilde{P}_{j}^{\text {loss }}\right)_{i}}{P_{0_{j}}}, & \text { otherwise }\end{cases}
$$


Remark 2: The conditions in (11) are based on the actual power consumption $P_{i}(t)=P_{0_{i}} \cdot \Pi_{i}^{P}(t)$. Let us define $P_{i}^{R_{\max }}=P_{0_{i}}\left(\Pi_{0}-\Pi_{\min }\right)_{i}$ and $P_{j}^{R_{\max }}=P_{0_{j}}\left(\Pi_{0}-\Pi_{\min }\right)_{j}$ as the maximum TCL response capability of busbar $i$ and of area $j$, respectively. Note that $P_{0_{i}}$ and $P_{0_{j}}$ are the steadystate power consumption of TCLs at busbar $i$ and area $j$. If the estimated infeed loss $\left(\tilde{P}_{j}^{l o s s}\right)_{i}$ by the TCLs at busbar $i$ is greater than the maximum response capability of the area $j$ in which they are located, they reduce their aggregate power up to the minimum level $P_{i}(t)=P_{0_{i}} \cdot \Pi_{\text {min }}$ (i.e. $\Pi_{i}^{P}=\Pi_{\text {min }}$ ). Otherwise, the TCLs at busbar $i$ share the burden with other TCLs in the same area (at different busbars) according to the their maximum response capability with respect to the area one. Accordingly:

$$
P_{i}(t)=P_{0_{i}}-\frac{P_{i}^{R_{\max }}}{P_{j}^{R_{\max }}}\left(\tilde{P}_{j}^{\text {loss }}\right)_{i}
$$

Substituting $P_{i}(t)=P_{0_{i}} \cdot \Pi_{i}^{P}(t)$ into (12), dividing by $P_{0_{i}}$ and solving for $\Pi_{i}^{P}(t)$, we obtain:

$$
\Pi_{i}^{P}(t)=\Pi_{0}-\frac{\left(\Pi_{0}-\Pi_{m i n}\right)_{i}}{P_{0_{j}}\left(\Pi_{0}-\Pi_{m i n}\right)_{j}}\left(\tilde{P}_{j}^{\text {loss }}\right)_{i}
$$

In case of homogenous TCLs, $\left(\Pi_{0}-\Pi_{\min }\right)_{i}=\left(\Pi_{0}-\right.$ $\left.\Pi_{\min }\right)_{j}$ and (13) are as in (11). The general case of heterogeneous devices does not affect the proposed framework.

Using semi-autonomous operation, $H_{j}$ and $P_{0_{j}}$ (and $\Pi_{m i n_{j}}$ for heterogeneous TCLs) could be updated according to the dispatch situation which would be done by the system operator or the demand aggregator. The broadcast of these values is not time critical. The inertia value $H_{j}$ depends on the generators dispatch and may vary over longer time scales (in the hours range). The steady-state power $P_{0_{j}}$ could be measured or derived from TCL parameters like duty cycles and size of TCL population. For simplicity, $P_{0_{j}}$ can be assumed time-invariant as a first approximation. Note that the TCLs provide maximum support over primary and secondary response time frame when $\Pi_{i}^{P}(t)=\Pi_{\min }$ in (11). Temperature constraints (4) are not binding even under this extreme scenario, as demonstrated in Section VII-A (Fig. 7).

After delivering secondary response (up to 30 minutes), the TCLs could recover to their steady-state power consumption level by means of a positive power-ramp. Depending on the speed at which the steady-state temperature condition should be regained, an extra-power consumption $\left(\Pi^{P}(t)>\Pi_{0}\right)$ could be implemented for a certain time. The optimal recovery and the amount of extra power absorbed could be decided based on several techno-economic considerations (e.g. units available for reserve). Optimizing the TCL response for longer time scales (above 30 minutes) is not within the scope of this study. Note that for the LC, the temperature constraint (4) is even less binding as $\Pi^{L}(t) \gg \Pi_{\text {min }}$ over the secondary response time window.

\section{Reduced Equivalent of the GB Power System}

A reduced dynamic equivalent model of the power system in Great Britain (GB) is considered for the case study. This model has been developed by the system operator, National
Grid (NG), for academic research [37]. The system is modeled in DIgSILENT PowerFactory software and the single-line diagram of the network is shown in Fig. 2.

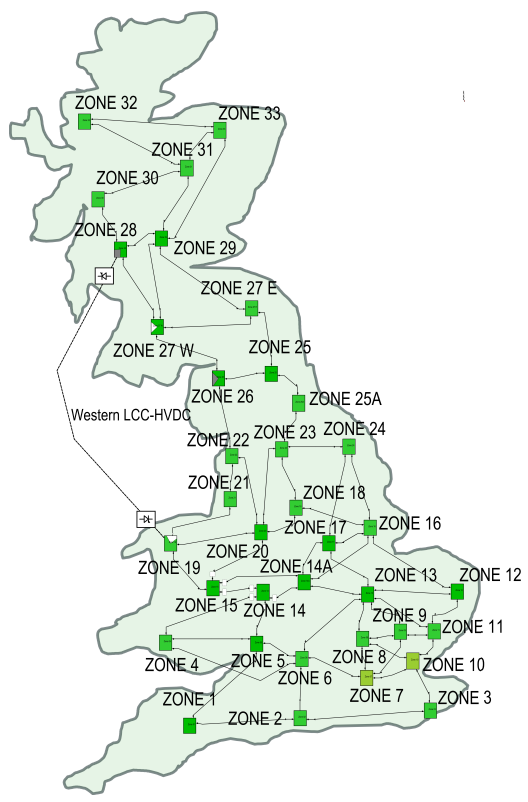

Fig. 2. Reduced dynamic equivalent of the Great Britain (GB) power system.

There are $64 \mathrm{AC}$ transmission lines at $400 \mathrm{kV}$ level, one embedded sub-sea DC link (Western HVDC) and a DC interconnector to Europe connected at busbar 12. The 36 busbars (designated as 'zones' in Fig. 2) represent the principal generation and demand centers. Different types of generators (coal, gas, hydro, biomass, nuclear and wind) within each geographical area are clubbed together at each busbar. The synchronous generators are equipped with standard models of governor control (except the nuclear power plants), automatic voltage regulator (AVR) and power system stabilizer (PSS). The governor control includes an integral term to reduce the steady-state speed (frequency) deviation to zero i.e. provide secondary response. The non-synchronous generators (NSGs) are represented by static models and do not provide any frequency service. Note that the spinning reserve is maintained at $1.8 \mathrm{GW}$ which is the largest possible infeed loss in GB for which NG has to ensure system security [29].

The overall demand is distributed at each busbar and is comprised of two types of aggregated loads. A 'static' load ( $P$-constant current, $Q$-constant impedance) and a 'TCL' load which is modeled as a controllable active power load according to the two different TCL control strategies (LC/PSSC) described previously. The total demand for the chosen operation scenario is $40 \mathrm{GW}$. About $40 \%$ of the demand $(16 \mathrm{GW})$ is supplied by wind farms and $2.5 \mathrm{GW}$ is imported through a DC interconnector. Under this situation, the total system inertia is 118.5 GW-s which is in line with the figures projected by NG [2].

Only domestic refrigerators with freezer compartment are considered as TCLs and are henceforth referred to as 'TCL'. The TCL parameters can be found in [34]. The total steadystate average power consumption $P_{0}$ of approximately 65 
million domestic refrigerators in GB is about $2.56 \mathrm{GW}$ (each refrigerator consumes $180 \mathrm{~W}$ ). Their minimum total power consumption $P_{\min }$ is $1.18 \mathrm{GW}$ according to (3).

\section{Simulation Results}

Simulation results are presented under two separate subsections to first demonstrate the effectiveness of TCLs for primary and secondary frequency response provision and then to establish the accuracy of the proposed infeed loss estimation algorithm for the pre-set shape controller (PSSC). The simulation study show a coherent system level TCL response with the proposed controllers despite the spatial variation of inertia.

The performance of the PSSC depends on the accuracy of infeed loss estimation which in turn relies on the identification of coherent areas in (9) as described in Section V-A. In this work, coherent generators were identified by examining the system dynamic response (measurement-based approach) to the different generator outages. More rigorous techniques (e.g. [38]) could be used but this is out of the scope of this work.

Based on the transient evolution of frequencies at different generators without response from the TCLs (as shown in Fig. 3) three distinct coherent groups of busbars or areas were identified. The outage event corresponds to the sudden disconnection of a $1.8 \mathrm{GW}$ generator located at busbar $27 \mathrm{E}$ at $t=2 \mathrm{~s}$. This outage amount is the largest possible infeed loss in the GB system at present [29] and therefore was chosen as the base case for the studies. Under the current dispatch scenario, other infeed loss disturbances would lead to similar (or simpler) identification of system areas.

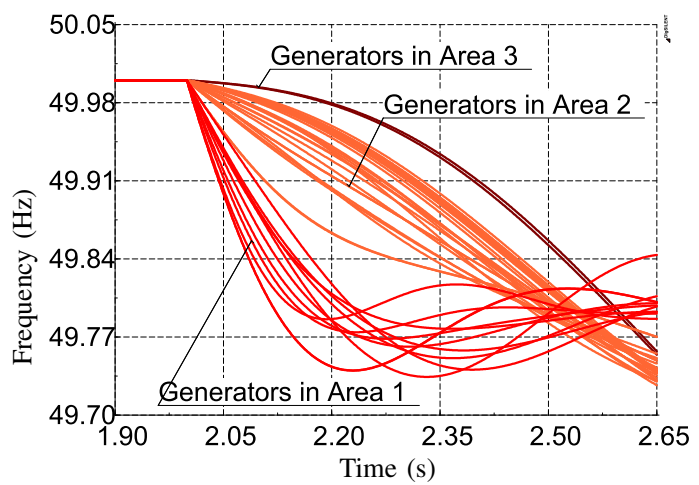

Fig. 3. Dynamic variation of frequencies measured at generator terminals.

Area 1 (red traces) includes all the generators in Scotland, in the north of the GB system (shown in Fig. 2). The rate of decay of frequency is faster in Area 1 where the infeed loss takes place and also because of low aggregate inertia. These frequencies differ from those in England and Wales (south of the system) due to relatively large reactance (electrically far) of the interconnection between Scotland and England/Wales. The generators in England/Wales are clustered into two coherent areas, Area 2 (orange traces) and Area 3 (brown traces) which includes only busbar 1 having two generators with large inertia and weak connection to the rest of the network. For infeed loss estimation, aggregated inertia of Areas 1,2 and 3 were found to be $H_{1}=16.2 \mathrm{GW} \cdot \mathrm{s}, H_{2}=73.9 \mathrm{GW} \cdot \mathrm{s}$ and $H_{3}=18.4 \mathrm{GW} \cdot \mathrm{s}$, respectively. The inertia of the generator causing the largest infeed loss were subtracted to find the aggregated inertia of Area 1 where it is located. For semi-autonomous operation, it is reasonable to consider worst (i.e. minimum) level of inertia for the TCLs located in the area where largest infeed loss is likely. The actual size and location of the event could affect the accuracy of the total infeed loss estimation for the PSSC as discussed later in detail in Section VII-B.

\section{A. Frequency Response from TCLs}

The operating scenario described in Section VI considers about $40 \%$ percent of the moderate demand level ( $40 \mathrm{GW})$ being met by non-synchronous generators (NSGs) (wind in this case). Under this situation, a sudden disconnection of a $1.8 \mathrm{GW}$ power plant located at busbar 27-E was simulated to create a frequency event. All the results presented in this subsection consider this above scenario and infeed loss event.

1) Benefits from TCL Primary Response (PR): Loss of $1.8 \mathrm{GW}$ generation at busbar 27-E leads to a frequency nadir below $49.2 \mathrm{~Hz}$ (as shown by the red traces in Fig. 4 (a)) which is not permissible in GB. This implies that the system operator would have to curtail part of the amount of wind generation to avoid this possibility of frequency problem. It is to be noted that this largest infeed loss does not result in RoCoF greater than the permissible limit $(1 \mathrm{~Hz} / \mathrm{s}$ using a $500 \mathrm{~ms}$ measuring window) anywhere in the system.

The green and blue traces in Fig. 4 (a) show the frequency nadirs for all busbars with TCL support using either the LC (green traces) or the PSSC (blue traces) are maintained well within the $49.2 \mathrm{~Hz}$ limit. Thus, in presence of TCL support, even more than $40 \%$ of the moderate demand could be supplied by the NSGs without worry for unacceptable frequency deviations. The PSSC yields improved frequency nadirs and relatively less deviation from the nominal frequency at the end of the primary response (PR) time frame. Variation of frequency with the LC is slightly worse (but still very much within acceptable limits) than that with the PSSC. This is due to negative RoCoF values during frequency recovery phases when the second term of (7), $K_{2} \cdot \operatorname{RoCoF}_{i}\left(t, \Delta t_{s}\right)$, counteracts the first term $K_{1} \cdot \Delta f_{i}(t)$ and reduces the TCL support. This counter effect diminishes to zero as soon as the frequency becomes steady.

2) Primary Response (PR) from Conventional Generators: With TCL support the need for PR from conventional generators reduces significantly (as shown in Fig. 4 (b)) compared to the reference case (without TCL support). The maximum PR required is reduced by about $920 \mathrm{MW}$ with the LC and 1710 MW with the PSSC. Thus, support from TCLs not only allows larger penetration of NSGs (i.e. RES) but also reduces (or even avoids) inefficient part-loaded operation of synchronous generators which is required to maintain the necessary PR reserve. This would clearly reduce the system operational cost.

3) Distributed TCL Response: The aggregate response of the TCLs (sum of all TCL responses at each busbar) in terms of overall reduction in power consumption from the nominal level is shown in Fig. 4 (c). The TCL power evolution at two selected busbars is plotted in Fig. 5. It is to be noted that the 

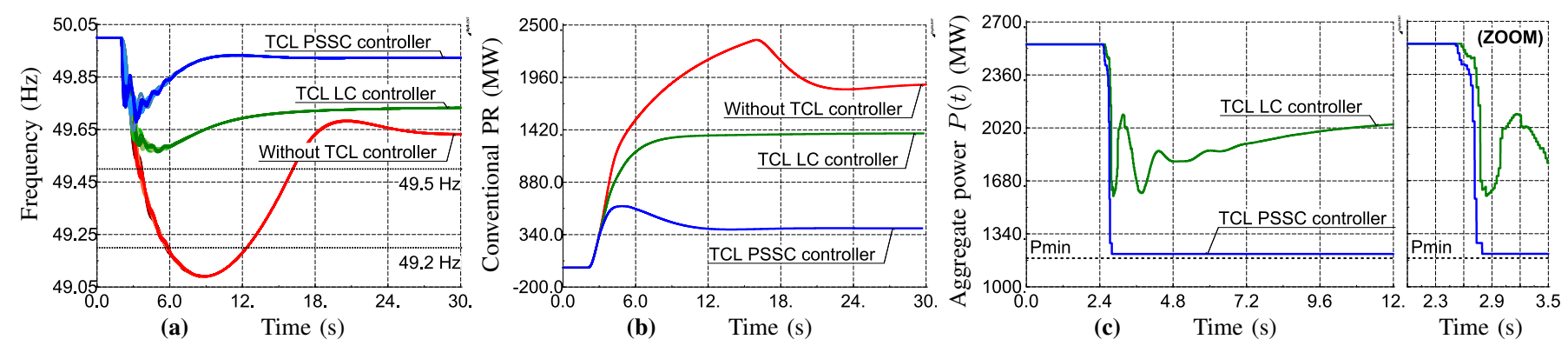

Fig. 4. System level performance during primary response (PR): (a) Dynamic variation of frequencies at all the system busbars with and without TCL support; (b) Total primary response (PR) from all conventional generators; (c) Overall reduction in power consumption of aggregate TCL response.

activation of TCL response is non-simultaneous due to the different activation times depending on the geographical location.

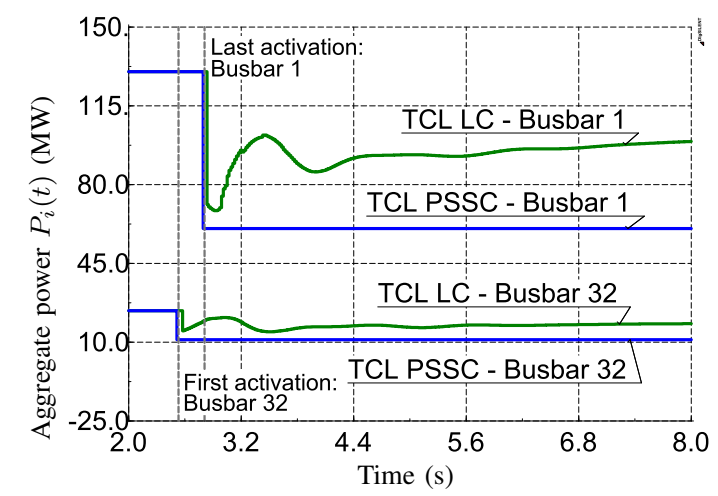

Fig. 5. Aggregate TCL response at busbar 1 and 32.

Focusing on the TCL overall response (Fig. 4 (c)), with the LC, TCLs maintain their maximum power reduction above the allowable limit $P_{\min }$ as the power consumption of individual TCL is reduced according to the locally measured frequency and RoCoF. Alternatively, for the PSSC, the minimum power threshold is reached for almost every busbar. Therefore, the aggregate power reduction is very close to the limit $P_{\min }$. For PR provision, the power reduction has to be achieved within 10 seconds after the detection of a frequency event (see Section III). A zoomed view of the TCL response for immediately after the infeed loss demonstrates both the LC and the PSSC are able to achieve the respective power reduction much faster within one second. This shows that TCLs can be a potential provider of the recently announced Enhanced Frequency Response (EFR) service [4], where the maximum allowed response time is one second. It is to be noted that the response time with the PSSC is slightly quicker than that with the LC. As both power profiles in Fig. 4 (c) respect the power/temperature constraints in (3) and (4), there is no need to simulate individual TCLs to ascertain the feasibility of aggregate the response [28].

4) Benefits from TCL Secondary Response (SR): Possible role of TCLs in proving secondary response (SR) is shown here. The restoration of frequencies back to the nominal value over a 30 minute period is shown in Fig. 6. Note that, besides conventional generators' support, secondary response is provided by TCLs according to the control strategies in (7) and (11). Use of the PSSC speeds up the frequency restoration process as it maintains the same level of TCL response as the
PR phase. The SR with the LC is much less (almost similar to the case with no TCL support) due to low TCL response driven by $\Delta f_{i}(t)$ and $\operatorname{RoCoF}_{i}\left(t, \Delta t_{s}\right)$ which are small and zero, respectively, at the end of the PR time frame. Similarly, the amount of power reserve and the associated ramp-rates required for conventional generators to provide SR reduce significantly in presence of TCL support using the PSSC.

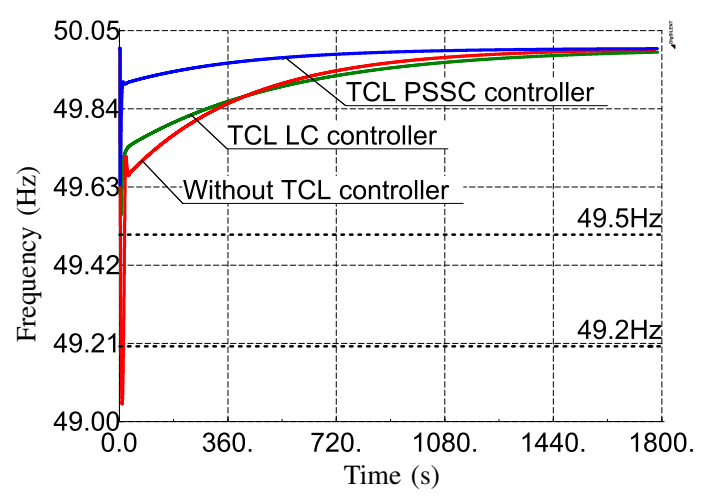

Fig. 6. Restoration of frequencies during secondary response (SR).

The evolution of the average temperature $\bar{T}$ associated to the aggregated TCL response for the LC and PSSC controllers during the SR period is shown in Fig. 7.

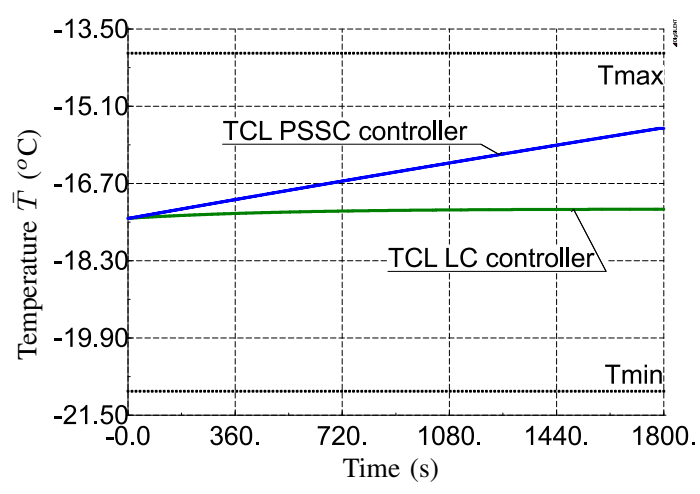

Fig. 7. Temperature evolution during secondary response (SR).

The temperature variation in both cases is well within boundary limits $\left[T_{\min }, T_{\max }\right]$, although, for the PSSC, a larger variation is observed. The more supporting system response for the PSSC seen in Fig. 6 comes at the expense of this larger temperature excursion. After the SR provision, a recovery phase is expected for the devices in which they would regain their previous operating status. 
5) Comparison with Alternative TCL controller: The performance of the proposed controllers (LC and PSSC) is now compared with an alternative TCL control strategy for frequency support provision, reported in [22]. This controller is chosen for comparison as it is described through a compact model (unlike other device-centric solutions), which facilitates its implementation in DIgSILENT. It features an average temperature variation proportional to frequency and an aggregated TCL response $P_{i}(t)$ according to two switching functions $\left(\lambda_{1}\right.$ and $\left.\lambda_{2}\right)$ which are temperature dependant. The controller parameters are as in [22]. Note that in this case the TCL response is also activated according to Section III-A. The result of this comparison is seen in Fig. 8 in terms of frequency variation during the PR period.

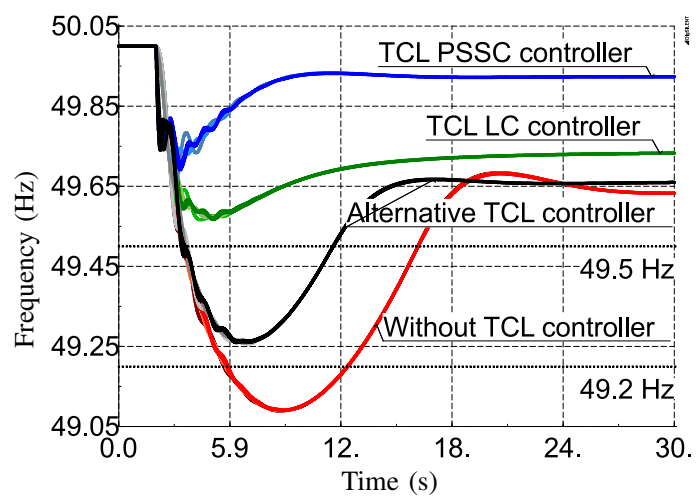

Fig. 8. Dynamic variation of frequencies at several system busbars for different TCL controllers.

For the same type of devices and parameters, the performance of this alternative controller (in black traces) is less effective than the presented LC and PSSC controllers in improving the frequency nadir. At post-fault steady-state, the frequency is very similar to the case without TCL controller. These results follow from a slow control over the TCL temperature distribution and its associated aggregate consumption performed by the controller. This comes from the fundamental idea to modulate the properties of the steady-state distribution.

\section{B. Infeed Loss Estimation for PSSC}

Alongside the $1.8 \mathrm{GW}$ infeed loss event mentioned in the previous subsection, two other events are considered here to validate the accuracy of the proposed infeed loss estimation algorithm and also to check the activation of the correct amount of TCL response. The two infeed loss events are 1) outage of a CCGT plant producing $594 \mathrm{MW}$ at busbar 10 and 2) outage of a $\mathrm{DC}$ interconnector importing $2.5 \mathrm{GW}$ into $\mathrm{GB}$ at busbar 12 .

1) Estimation Accuracy and TCL Response: For each of the three areas, the average estimated infeed loss across the busbars in each area $\tilde{P}_{j}^{\text {loss }}[\mathrm{MW}]$ and the corresponding TCL response in terms of their aggregate power reduction $P_{j}^{T C L}[\%]$ (expressed as percentage of the capability of the individual area $\left.\left(P_{0_{j}}-P_{j}(t)\right) / P_{j}^{R_{\max }}\right)$ are summarized in Table I for the three events mentioned above.

The estimated total infeed loss figures for the whole system $\tilde{P}_{\text {loss }}^{\text {sys }}$, shown on the rightmost column, are reasonably close to
TABLE I

INFEED LOSS ESTIMATION AND TCL RESPONSE

\begin{tabular}{|c|c|c|c|c|c|c|c|}
\hline \multirow[b]{2}{*}{$\begin{array}{l}P_{\text {loss }} \\
\text { [MW] }\end{array}$} & \multicolumn{2}{|c|}{ Area 1} & \multicolumn{2}{|c|}{ Area 2} & \multicolumn{2}{|c|}{ Area 3} & \multirow{2}{*}{$\begin{array}{l}\text { Total } \\
\tilde{P}_{s y s}^{\text {loss }} \\
{[\mathrm{MW}]}\end{array}$} \\
\hline & $\begin{array}{l}\tilde{P}_{1}^{\text {loss }} \\
{[\mathrm{MW}]}\end{array}$ & $\begin{array}{l}P_{1}^{T C L} \\
{[\%]}\end{array}$ & $\begin{array}{l}\tilde{P}_{2}^{\text {loss }} \\
{[\mathrm{MW}]}\end{array}$ & $\begin{array}{l}P_{2}^{T C L} \\
{[\%]}\end{array}$ & $\begin{array}{l}\tilde{P}_{3}^{\text {loss }} \\
{[\mathrm{MW}]}\end{array}$ & $\begin{array}{l}P_{1}^{T C L} \\
{[\%]}\end{array}$ & \\
\hline 594 & 101.6 & 100 & 382.9 & 32.9 & 76.7 & 57.7 & 561.2 \\
\hline 1800 & 247.7 & 100 & 1179.3 & 97.9 & 378.7 & 100 & 1805.7 \\
\hline 2500 & 355.1 & 100 & 1638.1 & 100 & 286.1 & 100 & 2279.3 \\
\hline
\end{tabular}

the actual infeed losses $P_{\text {loss }}$ (left most column). As discussed previously, for infeed loss calculations, each area relies on a set of aggregate inertia which are calculated considering the maximum credible infeed loss. This results in a difference between the actual and the estimated values.

The power consumption of TCLs is reduced according to the estimated area infeed loss at each busbar. The installed capacity of TCLs in Areas 1, 2 and 3 are 248.5, 2177.5 and 130.2 MW, respectively. Thus, about $38.7 \%, 98.2 \%$ and $100 \%$ of the total system-wide TCL capacity is used for the $594 \mathrm{MW}$, $1800 \mathrm{MW}$ and $2500 \mathrm{MW}$ infeed loss events, respectively. In Area 1 (Scotland), TCLs provide maximum (100\%) response regardless of the the size of the estimated infeed loss. This is due to relatively low inertia level and concentration of TCLs in the area. For Areas 2 and 3, TCL response is in proportion to the estimated infeed loss for the 594 MW infeed loss event due to higher regional inertia and more distributed TCL population. For the other two events, the TCL response is used in full (or almost). It can be seen from Fig. 9 that even for the $2.5 \mathrm{GW}$ infeed loss event, the frequency nadirs with TCL support are maintained above $49.5 \mathrm{~Hz}$. Without TCL support, the frequency nadirs would drop down to $48.8 \mathrm{~Hz}$ triggering dedicated demand disconnection plans [29]. The effectiveness of the proposed PSSC is more evident for smaller infeed loss events. The TCL response following the $594 \mathrm{MW}$ infeed loss event does not introduce any significant undesirable positive frequency excursion as shown in Fig. 9.

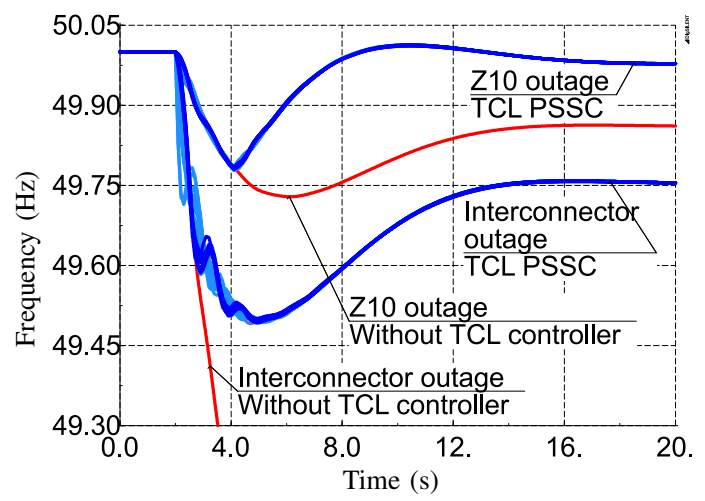

Fig. 9. Dynamic variation of frequencies for different infeed loss events: no TCL controller vs. TCL PSSC controller.

One major concern for low to moderate infeed loss events is the ability of TCL response to adapt without employing continuous updates of frequency measurements. This is adequately addressed with the proposed PSSC. Note that the average temperatures for the TCL population (after 30 minutes) are $-16.70^{\circ} \mathrm{C},-15.54^{\circ} \mathrm{C}$ and $-15.54^{\circ} \mathrm{C}$ for the $594 \mathrm{MW}, 1800 \mathrm{MW}$ 
and $2500 \mathrm{MW}$ infeed loss events, respectively. This is in compliance with the temperature limits established in (4).

2) Comparison with Alternative Methods: The performance of the proposed 'Area' method for infeed loss estimation is compared against two alternative approaches considering the 1.8 GW infeed loss event in Table II. A 'Busbar' approach uses the RoCoFs and the inertias at each busbar while a 'System' approach employs local RoCoFs but the system-wide aggregate inertia. The former estimates the proportion of the infeed loss at each busbar which are added up to derive the system-wide infeed loss estimate. In the latter, a system-wide infeed loss is estimated at each busbar and an average overall figure is used. All these estimation methods are compatible with the semi-autonomous operation of the PSSC.

The 'Busbar' method produces accurate estimate of overall infeed loss. However, the busbars with relatively low inertia would underestimate the size of the infeed loss despite severe local RoCoFs and thereby, activate only a fraction of the required (available) TCL response. For example, busbar 8 has the largest TCL population (nominal power consumption of $234 \mathrm{MW}$ ) but relatively small amount of inertia connected. Therefore, only $4 \mathrm{MW}$ out of the available $105 \mathrm{MW}$ of TCL response is activated at busbar 8 using the 'Busbar' method. This also evident from the low (39\%) overall activation of TCL response for the 'Busbar' method in Table II.

TABLE II

COMPARISON OF ESTIMATION ERROR AND TCL RESPONSE WITH TWO ALTERNATIVE INFEED LOSS ESTIMATION METHODS

\begin{tabular}{c|c|c}
\hline Method & Estimation error & Aggregate TCL response \\
\hline Busbar & $0.35 \%$ & $39.0 \%$ \\
Area & $0.31 \%$ & $98.2 \%$ \\
System & $5.74 \%$ & $100 \%$ \\
\hline
\end{tabular}

For the 'System' method, infeed loss is overestimated (maximum error of 53\%) at each busbar resulting in poor overall estimation as indicated by $5.74 \%$ error for 'System' method in Table II. The total TCL response is activated everywhere regardless of the size of the actual infeed loss. For smaller infeed loss events (e.g. the $594 \mathrm{MW}$ ), TCL response over-activation would cause undesirable positive frequency excursions. The proposed 'Area' method overcomes the above mentioned problems to achieve reasonably accurate infeed loss estimation and and at the same time activate the required TCL response as is clear from Table II. Using the 'Area' method some busbars in Area 2 (see Table I) estimate a generation loss (relative to that area) smaller than the capacity of the TCLs in that area (see (11)) and activate only the necessary amount of TCL response.

\section{LC Performance for Different Infeed Losses}

For the sake of completeness, the performance of the LC is also studied for the different loss of infeed outages analyzed in the previous subsection (594 MW and $2500 \mathrm{MW}$ ). The system response with this TCL controller is shown in Fig. 10 against the case in which the TCLs do not provide frequency support. It can be observed that, despite the fact of not updating $K_{1}$ and
$K_{2}$, the system response is well improved after the different outages. The control gains are chosen according to (8) to provide an effective response for different scenarios.

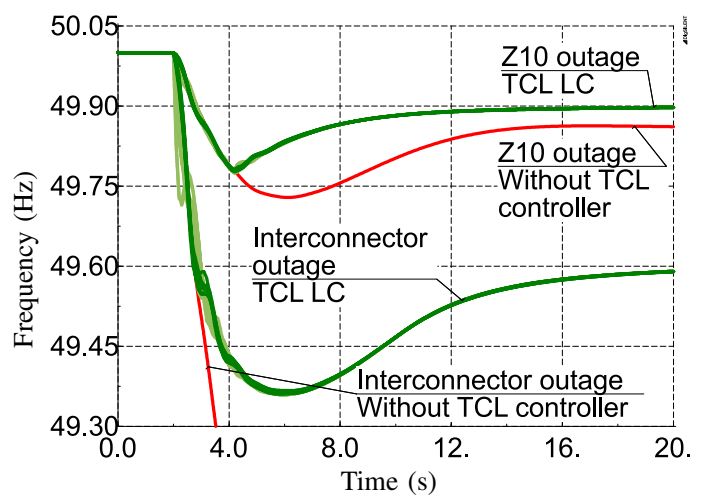

Fig. 10. Dynamic variation of frequencies for different infeed loss events: no TCL controller vs. TCL LC controller.

\section{CONCLUSIONS}

A realistic measure of the effectiveness of Thermostatically Controlled Loads (TCLs) in collectively contributing to grid frequency regulation has been demonstrated in this paper. Two TCL control strategies have been validated on a representative multi-machine reduced equivalent model of the GB power system, which captures the transient variation of system frequencies at the different busbars. This effect can be more pronounced in future low inertia scenarios. The TCLs (domestic refrigerators, in particular) were controlled without real-time communication and in a non-disruptive way for this purpose. Out of the two alternative control strategies for TCLs - Linear Controller (LC) and Pre-Set Shape Controller (PSSC) - the PSSC performs slightly better compared to the LC for primary response but produces significantly improved secondary response. However, a semi-autonomous operation is required for an effective performance of the PSSC. The LC, in contrast, due to the choice of $K_{1}$ and $K_{2}$, can adapt to different scenarios even under an autonomous operation. Note that, more advanced applications, which envisage the simultaneous provision of frequency response and energy arbitrage [34], would need a semi-autonomous operation for both controllers.

The studies reported in this paper demonstrate that, using either control strategy, rapid frequency response contribution (within one second) from TCLs can restrict the transient frequency deviation well within the acceptable $49.2 \mathrm{~Hz}$ limit following large infeed losses. As a consequence, the amount of frequency response services required from conventional generators is drastically reduced. This would allow the system operator to accommodate larger shares of RES (wind etc.) without having to worry about curtailing those to retain adequate synchronous generation (and hence adequate inertia and conventional response) in the system. Instead, without any TCL support, the system frequency would drop below the minimum threshold. The results also demonstrate that TCLs are potential candidates to provide Enhanced Frequency Response (EFR), as their support is delivered within one second. 
The proposed 'Area' infeed loss estimation method achieves a reasonable accuracy and ensures activation of the required TCL distributed response which is not possible with a 'Busbar' or 'System' level approach. This is critical for the PSSC to avoid over- or under-activation of TCL response and the consequent negative impact on frequency regulation.

It must be acknowledged that the present study and proposed TCL control strategies for frequency support provision are derived assuming a first-order linear model to represent the TCL temperature dynamics. Introducing more complex TCL models into the control framework to account, for instance, for the time delays introduced by the compressor in some type of devices, is being pursued by our research group [39].

Our ongoing work is also focused on analyzing the performance of the proposed controllers for more severe scenarios (lower inertia, larger infeed loss) which could lead to the activation of RoCoF sensitive protection schemes of embedded generators. Among other factors, the activation settings of the TCLs would be crucial to ensure secure dynamic response under such extreme scenarios. In addition, the effects of the TCL response at the distribution level (considering a distribution feeder in the power system model) are to be studied.

\section{ACKNOWLEDGMENT}

The authors would like to thank Simon Tindemans for his support on device-level simulations and the anonymous reviewers for their helpful comments on the manuscript.

\section{REFERENCES}

[1] National Grid. Mandatory Frequency Response. [Online]. Available: http://www2.nationalgrid.com/uk/services/balancing-services/ frequency-response/mandatory-frequency-response/

[2] —. (2015) System Operability Framework (SOF). [Online]. Available: http://www2.nationalgrid.com/UK/Industry-information/ Future-of-Energy/System-Operability-Framework/

[3] G. Lalor, A. Mullane, and M. O'Malley, "Frequency control and wind turbine technologies," Power Systems, IEEE Trans. on, vol. 20, no. 4, pp. 1905-1913, Nov 2005.

[4] National Grid. (2015) Enhanced Frequency Response. [Online]. Available: http://www2.nationalgrid.com/Enhanced-Frequency-Response. aspx

[5] Z. Akhtar, B. Chaudhuri, and S. Y. R. Hui, "Primary frequency control contribution from smart loads using reactive compensation," IEEE Transactions on Smart Grid, vol. 6, no. 5, pp. 2356-2365, Sept 2015.

[6] Y. Q. Bao, Y. Li, Y. Y. Hong, and B. Wang, "Design of a hybrid hierarchical demand response control scheme for the frequency control," IET Generation, Transmission Distribution, vol. 9, no. 15, pp. 23032310, 2015.

[7] M. R. V. Moghadam, R. T. B. Ma, and R. Zhang, "Distributed frequency control in smart grids via randomized demand response," vol. 5, no. 6 , Nov 2014, pp. 2798-2809.

[8] M. Donnelly, D. Harvey, R. Munson, and D. Trudnowski, "Frequency and stability control using decentralized intelligent loads: Benefits and pitfalls," in PES General Meeting, 2010 IEEE, Jul 2010, pp. 1-6.

[9] L. Jia and L. Tong, "Dynamic pricing and distributed energy management for demand response," IEEE Transactions on Smart Grid, vol. 7 , no. 2, pp. 1128-1136, March 2016.

[10] D. Papadaskalopoulos and G. Strbac, "Decentralized participation of flexible demand in electricity markets - part I: Market mechanism," IEEE Transactions on Power Systems, vol. 28, no. 4, pp. 3658-3666, Nov 2013.

[11] M. Aunedi, P. A. Kountouriotis, J. E. O. Calderon, D. Angeli, and G. Strbac, "Economic and environmental benefits of dynamic demand in providing frequency regulation," IEEE Transactions on Smart Grid, vol. 4, no. 4, pp. 2036-2048, Dec 2013.
[12] D. S. Callaway and I. A. Hiskens, "Achieving controllability of electric loads," Proceedings of the IEEE, vol. 99, no. 1, pp. 184-199, Jan 2011.

[13] ENTSO-E. (2015) Demand Connection Code. [Online]. Available: https://www.entsoe.eu/major-projects/network-code-development/ demand-connection

[14] J. Short, D. Infield, and L. Freris, "Stabilization of grid frequency through dynamic demand control," Power Systems, IEEE Trans. on, vol. 22, no. 3, pp. 1284-1293, Aug 2007.

[15] A. Molina-García, F. Bouffard, and D. S. Kirschen, "Decentralized demand-side contribution to primary frequency control," IEEE Transactions on Power Systems, vol. 26, no. 1, pp. 411-419, Feb 2011.

[16] V. Trovato, S. Tindemans, and G. Strbac, "Controlling the synchronization and payback associated with the provision of frequency services by dynamic demand," in Electricity Distribution (CIRED 2013), 22nd International Conference and Exhibition on, Jun 2013, pp. 1-4.

[17] K. Samarakoon, J. Ekanayake, and N. Jenkins, "Investigation of domestic load control to provide primary frequency response using smart meters," IEEE Transactions on Smart Grid, vol. 3, no. 1, pp. 282-292, March 2012.

[18] A. Molina-García, I. Muñoz-Benavente, A. D. Hansen, and E. GómezLázaro, "Demand-side contribution to primary frequency control with wind farm auxiliary control," IEEE Transactions on Power Systems, vol. 29, no. 5, pp. 2391-2399, Sept 2014

[19] M. Cheng, J. Wu, S. Galsworthy, N. Jenkins, and W. Hung, "Availability of load to provide frequency response in the Great Britain power system," in Power Systems Computation Conference (PSCC), 2014, Aug 2014, pp. 1-7.

[20] E. Ciapessoni, D. Cirio, F. Conte, S. Massucco, and F. Silvestro, "Demand side response for frequency control in a regional power system," in Clean Electrical Power (ICCEP), 2015 International Conference on, June 2015, pp. 258-264.

[21] M. Cheng, J. Wu, S. J. Galsworthy, C. E. Ugalde-Loo, N. Gargov, W. W. Hung, and N. Jenkins, "Power system frequency response from the control of bitumen tanks," IEEE Transactions on Power Systems, vol. 31, no. 3, pp. 1769-1778, May 2016.

[22] D. Angeli and P.-A. Kountouriotis, "A stochastic approach to "dynamicdemand" refrigerator control," Control Systems Technology, IEEE Trans. on, vol. 20, no. 3, pp. 581-592, May 2012.

[23] S. Weckx, R. D'Hulst, and J. Driesen, "Primary and secondary frequency support by a multi-agent demand control system," IEEE Transactions on Power Systems, vol. 30, no. 3, pp. 1394-1404, May 2015.

[24] J. L. Mathieu, M. Kamgarpour, J. Lygeros, G. Andersson, and D. S. Callaway, "Arbitraging intraday wholesale energy market prices with aggregations of thermostatic loads," IEEE Transactions on Power Systems, vol. 30, no. 2, pp. 763-772, March 2015.

[25] H. Hao, B. Sanandaji, K. Poolla, and T. Vincent, "Aggregate flexibility of thermostatically controlled loads," Power Systems, IEEE Trans. on, vol. 30, no. 1, pp. 189-198, Jan 2015.

[26] M. Vanouni and N. Lu, "Improving the centralized control of thermostatically controlled appliances by obtaining the right information," Smart Grid, IEEE Trans. on, vol. 6, no. 2, pp. 946-948, Mar 2015.

[27] C. Perfumo, E. Kofman, J. H. Braslavsky, and J. K. Ward, "Load management: Model-based control of aggregate power for populations of thermostatically controlled loads," Energy Conversion and Management, vol. 55 , pp. $36-48,2012$.

[28] S. Tindemans, V. Trovato, and G. Strbac, "Decentralized control of thermostatic loads for flexible demand response," Control Systems Technology, IEEE Trans. on, vol. 23, no. 5, pp. 1685-1700, Sep 2015.

[29] National Grid. (2012) NETS Security and Quality of Supply Standard, version 2.2. [Online]. Available: http://www2.nationalgrid.com/UK/ Industry-information/Electricity-codes/SQSS/The-SQSS/

[30] Ofgem. (2014) Changes to the Distribution Code and Engineering Recommendation G59: Frequencey Changes during Large Disturbances and their Impact on the Total System. [Online]. Available: https: //www.ofgem.gov.uk/publications-and-updates/

[31] P. Ashton, C. Saunders, G. Taylor, A. Carter, and M. Bradley, "Inertia estimation of the GB power system using synchrophasor measurements," Power Systems, IEEE Trans. on, vol. 30, no. 2, pp. 701-709, Mar 2015.

[32] F. Dörfler, M. Chertkov, and F. Bullo, "Synchronization in complex oscillator networks and smart grids," Proceedings of the National Academy of Science, vol. 110, no. 6, Nov 2012.

[33] V. Trovato, S. Tindemans, and G. Strbac, "Designing effective frequency response patterns for flexible thermostatic loads," in EEEIC 2015 IEEE 15th International Conference on, Jun 2015, pp. 1003-1008.

[34] — , "Leaky storage model for optimal multi-service allocation of thermostatic loads," IET Generation, Transmission \& Distribution, accepted - in press, Oct 2015. 
[35] DIgSILENT GmbH, "DIgSILENT PowerFactory, Version 15.1, User Manual," 2013.

[36] UK Government - DECC. (2014) Smart Metering Implementation Programme.

[37] H. Urdal, R. Ierna, J. Zhu, C. Ivanov, A. Dahresobh, and D. Rostom, "System strength considerations in a converter dominated power system," Renewable Power Generation, IET, vol. 9, no. 1, pp. 10-17, 2015.

[38] A. M. Khalil and R. Iravani, "A dynamic coherency identification method based on frequency deviation signals," IEEE Transactions on Power Systems, vol. 31, no. 3, pp. 1779-1787, May 2016.

[39] S. Tindemans and G. Strbac, "Nondisruptive decentralized control of thermal loads with second order thermal models," in PES General Meeting, 2016 IEEE, Jul 2016, pp. 1-5.

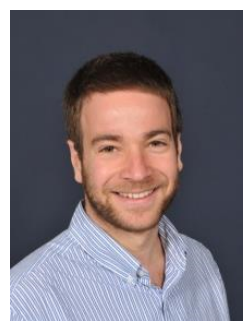

Vincenzo Trovato (M'15) was born in Bitonto, Italy. $\mathrm{He}$ is a Research Engineer at the EDF R\&D U.K. Centre and a Visiting Researcher at Imperial College London, London, U.K. Previously he was a Research Associate at Imperial College London where he obtained the Ph.D. degree in Electrical Engineering. He also owns a M.Eng Degree in Electrical Engineering from Politecnico di Torino, Italy. His research interests include demand side response and its impact on power system control and economics.

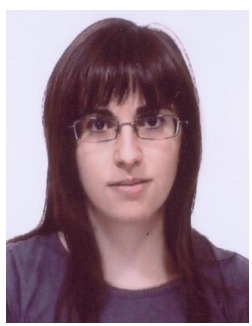

Inmaculada Martínez Sanz (S'13 - M'15) received the degree in Industrial Engineering from Universidad de Sevilla, Seville, Spain, in 2010 and the Ph.D. degree in Electrical and Electronic Engineering from Imperial College London, London, U.K., in 2015. She is currently a Research Associate at Imperial College London. Her research interests include power system dynamics and control, HVDC transmission and wind power.

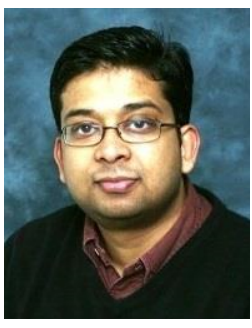

Balarko Chaudhuri (M'06 - SM'11) received the $\mathrm{Ph} . \mathrm{D}$. degree in Electrical and Electronic engineering from Imperial College London, London, U.K., in 2005 where he is currently a Senior Lecturer at the Control and Power Research Group. His research interests include power systems stability, grid integration of renewables, HVDC, FACTS, demand response and smart grids. Dr Chaudhuri is an editor of the IEEE Transactions on Smart Grid and an associate editor of the IEEE Systems Journal and Elsevier Control Engineering Practice. He is a Fellow of the Institution of Engineering and Technology (IET) and a member of the International Council on Large Electric Systems (CIGRÉ).

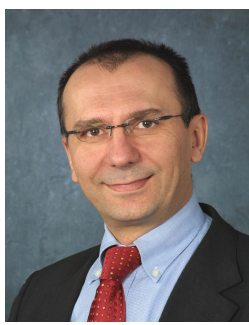

Goran Strbac (M'95) is a Professor of Energy Systems at Imperial College London, London U.K. His current research is in modeling and optimization of economics and security of energy system operation and investment including integration of emerging technologies in supporting cost effective evolution to smart low carbon energy future. 